\title{
RECYCLABLE ACIDIC BRØNSTED IONIC LIQUID CATALYZED SYNTHESIS OF QUINOXALINE
}

\author{
TEJESWARARAO D*a
}

${ }^{a}$ Indian Institute of Chemical Technolgy, Fine Chemical Laboratory, Tarnaka, Hyderabad, India.

\begin{abstract}
The acidic ionic liquid, 1-(4-sulfonic acid) butyl-3-methylimidazolium hydrogen sulfate [ $\left.\mathrm{MimC}_{4} \mathrm{SO}_{3} \mathrm{H}\right] \mathrm{HSO}_{4}$ catalyzed two-component condensation reaction of phenacyl bromide and $o$-phenylenediamine to afford corresponding quinoxaline derivatives. The inexpensive and non-toxic ionic liquids can be reused several times without any perceptible loss of their activities.
\end{abstract}

Keywords: Phenacyl bromide, Quinoxaline, Ionic liquids, Catalyst, Green chemistry.

\section{INTRODUCTION}

Quinoxalines, as a core structural motif, are everywhere in natural and unnatural compounds. In $20^{\text {th }}$ century the biological applications of diverse quinoxaline analogues has drawn the attention of many organic chemists. ${ }^{1-4}$ Quinoxaline and its derivatives play an important role in medicinal chemistry and new drug discovery. ${ }^{5}$ In addition to that these are having many applications in dyes preparation, chemically controllable switches, efficient electroluminescent materials, dehydroannulenes, and inorganic semiconductors. ${ }^{6-11}$ Quinoxaline ring moiety comprise part of the chemical structures of various antibiotics such as Echinomycin, Levomycin and Actinoleutin ${ }^{12,13}$, that are known to inhibit growth of gram positive bacteria and are also active against various transplantable tumors.

In recent literature a number of methods have been reported for the synthesis of substituted quinoxalines: oxidation-trapping of $\alpha$-hydroxy ketones with 1,2-diamines, ${ }^{14}$ 1,4-addition of 1,2-diamines to diazenylbutenes, ${ }^{15}$ cyclization-oxidation of phenacyl bromides and $o$-phenylenediamines through solid-phase synthesis ${ }^{16}$ and oxidative coupling of epoxides with ene-1,2diamines. ${ }^{17}$ 2,3-Disubstituted quinoxalines have also been prepared by SuzukiMiyaura coupling reaction, ${ }^{18}$ condensation of $o$-phenylenediamines and 1,2-dicarbonyl compounds in $\mathrm{MeOH} / \mathrm{AcOH}$ under microwave irradiation ${ }^{19}$. Most of the methods that have been reported for the synthesis of quinoxalines require elevated temperatures and complex catalysts. Hence, there is a need to develop a simple, eco-friendly method under mild conditions for the preparation of quinoxalines.

From last two decades ionic liquids have emerged as solvent and catalytic system in many organic transformations and preparation of inorganic compounds ${ }^{20,21}$, because of it's low vapour pressure, high viscosity, high conductivity, thermal and oxidative stability, and inflammability. In addition to that ionic liquids act as ideal medium in some specific reactions which involve reactive ionic intermediates. Furthermore, due to the stabilisation of charged reactive intermediates by ionic liquids, can promote high selectivity and rate of reaction and are finding broad applications in synthetic organic chemistry.

Herein we report the use of ionic liquid (Fig. 1) as recyclable solvent for the oxidative condensation of phenacyl bromide and $o$-phenylenediamine to produce quinoxaline derivatives in excellent yield at $65^{\circ} \mathrm{C}-75^{\circ} \mathrm{C}$ (Scheme 1).

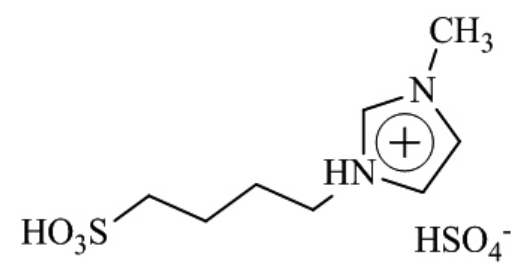

1-(4-sulfonic acid) butyl-3-methylimidazolium hydrogen sulfate $\left(\left[\mathrm{MimC}_{4} \mathrm{SO}_{3} \mathrm{H}\right] \mathrm{HSO}_{4}\right)$

Figure 1: Chemical structure of representative ionic liquid<smiles>O=C(CBr)c1ccccc1</smiles><smiles>Nc1ccccc1N</smiles>

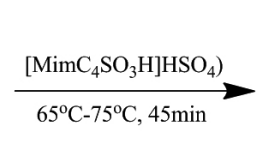<smiles>c1ccc(-c2cnc3ccccc3n2)cc1</smiles>

Scheme 1

All the reactions proceeded smoothly in ionic liquid without any need of other catalyst. In these reactions ionic liquid plays a dual role as solvent and catalyst. The reaction of 2-bromo-1-phenylethanone 1a with benzene1,2-diamine 2a in 1-(4-sulfonic acid) butyl-3-methylimidazolium hydrogen sulfate $\left(\left[\mathrm{MimC}_{4} \mathrm{SO}_{3} \mathrm{H}\right] \mathrm{HSO}_{4}\right)$ at $65-75^{\circ} \mathrm{C}$ over a period of $45 \mathrm{~min}$ resulted in the formation of 2 -phenylquinoxaline 3a in $95 \%$ yield. The product was isolated by simple extraction with diethyl ether. The left over viscous ionic liquid was thoroughly washed with ether and recycled in subsequent reactions. Encouraged by the satisfactory results of our first attempts, we examined several other phenacyl bromides. The scope and generality of this process is illustrated with respect to various aryl diamines and phenacyl bromides, and the results are presented in Table 1. Better yields were obtained when phenacyl bromide has electron donating substituents than electron withdrawing substituents, In all the cases excellent yields were obtained. In similar manner 2-bromo-1(6-chloropyridin-3-yl)ethanone condensed with various o-phenylenediamine derivatives to produce corresponding products (entries 12,13,14). 1-(4-sulfonic acid) butyl-3-methylimidazolium hydrogen sulfate $\left[\mathrm{MimC}_{4} \mathrm{SO}_{3} \mathrm{H}\right] \mathrm{HSO}_{4}$ and all phenacyl bromide derivatives were prepared according to reported procedures. ${ }^{22}$ This method is even effective with highly functionalized phenacyl bromides. For example, reaction of 2-bromo-1-(4-(3-fluoro-4-nitrophenoxy)phenyl) ethanone1i with $o$-phenylenediamine 2a under similar conditions to produce 2-(4-(3-fluoro-4-nitrophenoxy)phenyl) quinoxaline $\mathbf{3 o}$ in good yield (Scheme 2).

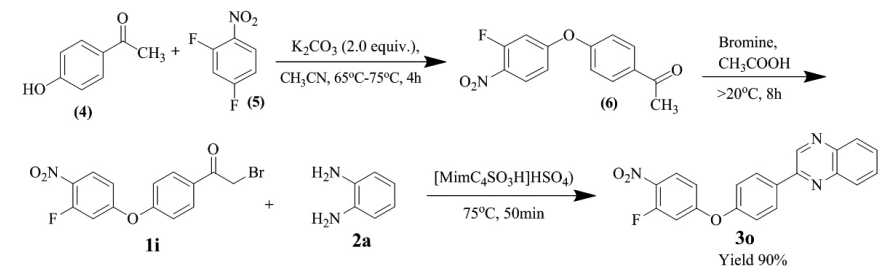

Scheme 2

aReaction conditions: Phenacyl bromide (1.0 mmol), o-phenylene diamine $(1.1 \mathrm{mmol})$, [MimC $\mathrm{SO}_{3} \mathrm{H} \mathrm{HSO}_{4}(5 \mathrm{~mol} \%)$. ${ }^{6}$ Isolated yield.

It is very important to recycle ionic liquids in the context of economic feasibility. In view of the efficiency of the reaction in $\left[\mathrm{MimC}_{4} \mathrm{SO}_{3} \mathrm{H}\right] \mathrm{HSO}_{4}$, we examined its recyclability. After separating the organic phase from the worked up reaction mixture, the left over $\left[\mathrm{MimC}_{4} \mathrm{SO}_{3} \mathrm{H}\right] \mathrm{HSO}_{4}$ was reactivated by drying under vacuum at $80-100^{\circ} \mathrm{C}$ for $20 \mathrm{~min}$., and was reused for the same reaction without any perceptible loss of catalytic activity. These results are shown in Figure 2. The yield was only slightly decreased after the third cycle, indicating that the catalyst possessed an excellent reusability under the same 
reaction conditions. Apart from yield and recyclability, this process involved simple work-up procedure for isolation of the desired final product.

Table 1: Synthesis of quinoxalines in-(4-sulfonic acid) butyl-3methylimidazolium hydrogen sulfate ${ }^{\mathrm{a}}$
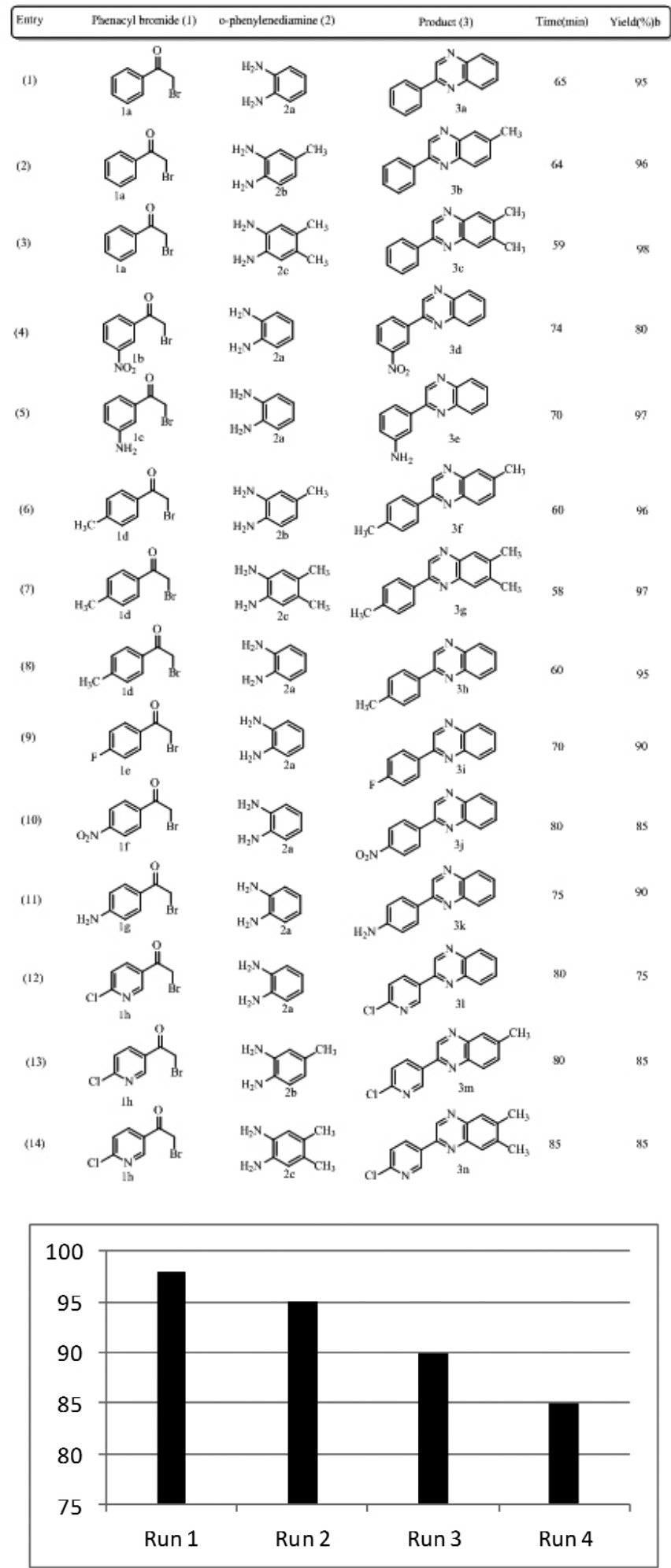

Figure 2: Graphical representation of obtained yields over four cycles
$\alpha$-Halocarbonyl compounds show better reactivity in the presence of ionic liquids thereby reducing the reaction times, and improving the yields. The rate enhancement in an ionic liquid is probably due to increased polarization of the $-\mathrm{CH}_{2}-\mathrm{Br}$ bond in the polar ionic medium and also because of stabilization of the charged ionic intermediates by ionic liquid. In an ionic liquid, polarization of carbonyl compound is increased significantly compared to organic solvents.

The experimental procedure is very simple and convenient in addition to that, it does not require any aqueous work-up thereby avoiding the generation of toxic waste. Since the products were weakly soluble in the ionic liquid, they were easily separated by simple extraction with ether. The rest of the viscous ionic liquid was thoroughly washed with ether and recycled. The recovered ionic liquid was activated at $85^{\circ} \mathrm{C}$ under reduced pressure and reused without any significant loss of activity. By using recovered ionic liquid in the second run afforded similar yield to that obtained in the first run. However, in the third and fourth run, the yields were gradually decreased. For example, the reaction of 2-bromo-1-(p-tolyl)ethanone 1d and benzene-1,2-diamine 2a in $\left[\mathrm{MimC}_{4} \mathrm{SO}_{3} \mathrm{H}\right] \mathrm{HSO}_{4}$ to produce 2-p-tolylquinoxaline $\mathbf{3 h}$ gave $95,93,92$, and $90 \%$ yields respectively over four cycles (Figure 1). The products obtained were of the same purity as in the first run. The simple experimental and product isolation procedures combined with ease of recovery and reuse of this novel reaction media is expected to contribute to the development of green strategies for the synthesis of quinoxalines.

In summary, this paper describes a convenient and efficient method for the synthesis of quinoxalines via the oxidative cyclization of highly reactive phenacyl bromides and $o$-phenylenediamine using ionic liquids as promoters. The simple experimental and product isolation procedures combined with ease of recovery and reuse of this reaction media is expected to contribute to the development of environmentally friendly processes for the synthesis of quinoxalines of biological and medicinal importance. The use of ionic liquids as promoters for this transformation avoids the use of moisture sensitive and heavy metal Lewis acids.

\section{EXPERIMENTAL}

SYNTHESIS AND CHARACTERIZATION OF (1-(4-SULFONIC ACID) BUTYL-3-METHYLIMIDAZOLIUM HYDROGEN SULFATE

1-Methylimidazole $(15.8 \mathrm{ml}, 0.2 \mathrm{~mol})$ and 1,4-butane sultone $(22 \mathrm{ml}$, $0.2 \mathrm{~mol}$ ) were charged into a $100 \mathrm{ml}$ round-bottom flask. Then, the mixture was stirred at $40^{\circ} \mathrm{C}$ for $10 \mathrm{~h}$. The white solid zwitterion was washed repeatedly with ether $(80 \times 5 \mathrm{ml})$ to remove non-ionic residues and dried in vacuum. Then, a stoichiometric amount of concentrated sulfuricacid $(98 \%, 10.9 \mathrm{ml})$ was added by dropwise and the mixture stirred for $6 \mathrm{~h}$ at $80^{\circ} \mathrm{C}$ to form the IL. ${ }^{1} \mathrm{H}$ NMR (400 MHz, $\left.\mathrm{D}_{2} \mathrm{O}\right): \delta=1.28-1.32\left(\mathrm{~m}, 2 \mathrm{H},-\mathrm{CH}_{2}\right), 1.56-1.60(\mathrm{~m}, 2 \mathrm{H}$, $\left.-\mathrm{CH}_{2}\right), 2.48-2.52\left(\mathrm{t}, J=7.7 \mathrm{~Hz}, 2 \mathrm{H},-\mathrm{CH}_{2}\right), 3.45\left(\mathrm{~s}, 3 \mathrm{H},-\mathrm{CH}_{3}\right), 3.78-3.82(\mathrm{t}, J=$ $\left.7.1 \mathrm{~Hz}, 2 \mathrm{H},-\mathrm{CH}_{2}\right), 7.00(\mathrm{~s}, 1 \mathrm{H}$, imidazole), $7.05(\mathrm{~s}, 1 \mathrm{H}$, imidazole), $8.27(\mathrm{~s}, 1 \mathrm{H}$, imidazole) $;{ }^{13} \mathrm{C}$ NMR $\left(100 \mathrm{MHz}, \mathrm{D}_{2} \mathrm{O}\right): \delta=20.75,27.90\left(-\mathrm{CH}_{2}\right), 35.63\left(-\mathrm{CH}_{3}\right.$, imidazole), 48.75, $49.94\left(-\mathrm{CH}_{2}\right), 121.90,123.62$ and 135.77 (-CH, imidazole).

General Procedure:

A mixture of phenacyl bromide $(1 \mathrm{mmol})$ and o-phenylenediamine $(1.2$ mmol) in $\left[\mathrm{MimC}_{4} \mathrm{SO}_{3} \mathrm{H}\right] \mathrm{HSO}_{4}(3 \mathrm{~mL})$ was heated at $70^{\circ} \mathrm{C}$ for the appropriate time (see Table 1). After completion of the reaction, as indicated by TLC, the reaction mixture was washed with diethylether $(3 \times 10 \mathrm{~mL})$. diluted with water $(3 \mathrm{ml} \times 10 \mathrm{ml})$. The combined ether extracts were concentrated in vacuo and the resulting product was directly charged onto a small silica gel column and eluted with a mixture of ethyl acetate:n-hexane $(0.5: 9.5)$ to afford pure quinoxaline. The rest of the viscous ionic liquid was further washed with ether and recycled in subsequent runs. However, in the case of solids, the products were purified by recrystallization from appropriate solvents.

The spectral and analytical data of all compounds are given below.

(3a) 2-phenylquinoxaline: ${ }^{15}$ Milky white colour solid; mp $75-78^{\circ} \mathrm{C} ;{ }^{1} \mathrm{H}$ NMR (300 MHz, $\left.\mathrm{CDCl}_{3}\right) \delta(\mathrm{ppm})$ 7.61-7.40 (m, 3H, Ar-H), 7.82-7.66 (m, 2H, Ar-H), 8.16-8.06 (m, 2H, Ar-H), 8.25-8.17 (m, 2H, Ar-H), 9.31 (s, 1H, Ar-H); ${ }^{13} \mathrm{C}$ NMR $\left(75 \mathrm{MHz}, \mathrm{CDCl}_{3}\right) \delta(\mathrm{ppm}) 127.3,129.0,129.1,129.5,129.6,130.1$, 130.2, 136.7, 141.5, 142.2,143.3, 151.7; MS (ESI) $\mathrm{m} / \mathrm{z} 207(\mathrm{M}+\mathrm{H})^{+}$; HRMS (ESI) Calcd for $\mathrm{C}_{14} \mathrm{H}_{10} \mathrm{~N}_{2}(\mathrm{M}+\mathrm{H})^{+}$207.0922, found 207.0929.

(3b) 6-methyl-2-phenylquinoxaline: White colour solid; mp $134-136^{\circ} \mathrm{C}$; ${ }^{1} \mathrm{H}$ NMR $\left(300 \mathrm{MHz}, \mathrm{CDCl}_{3}\right) \delta(\mathrm{ppm}) 2.62\left(\mathrm{~s}, 3 \mathrm{H}, \mathrm{Ar}_{-} \mathrm{CH}_{3}\right), 7.61-7.42(\mathrm{~m}, 4 \mathrm{H}$, Ar-H), 8.03-7.82 (m, 2H, Ar-H), 8.18 (m, 2H, Ar-H), 9.24 (d, $J=7.3 \mathrm{MHz}, 1 \mathrm{H}$, $\mathrm{Ar}-\mathrm{H}) ;{ }^{13} \mathrm{C}$ NMR $(75 \mathrm{MHz}, \mathrm{CDCl}) \delta(\mathrm{ppm}) 20.1,127.2,127.9,128.4,128.8$, $129.6,136.9,139.9,140.3,140.6,140.9,142.2,150.8 ; \mathrm{MS}(\mathrm{ESI}) \mathrm{m} / \mathrm{z} 220$ $(\mathrm{M}+\mathrm{H})^{+}$; HRMS (ESI) Calcd for $\mathrm{C}_{15} \mathrm{H}_{12} \mathrm{~N}_{2}(\mathrm{M}+\mathrm{H})^{+} 220.1082$, found 220.1085 .

(3c) 6,7-dimethyl-2-phenylquinoxaline: Light yellow colour solid; $\mathrm{mp}$ $122-124^{\circ} \mathrm{C} ;{ }^{1} \mathrm{H}$ NMR $\left(300 \mathrm{MHz}, \mathrm{CDCl}_{3}\right) \delta(\mathrm{ppm}) 2.51$ (s, 6H, Ar- $\left.\mathrm{CH}_{3}\right), 7.56-$ 
7.41 (m, 3H, Ar-H), 7.89-7.79 (m, 2H, Ar-H), 8.20-8.12 (m, 2H, Ar-H), 9.19 (s, $1 \mathrm{H}, \mathrm{Ar}-\mathrm{H}) ;{ }^{13} \mathrm{C} \mathrm{NMR}\left(\mathrm{CDCl}_{3}, 75 \mathrm{MHz}\right) \delta$ (ppm) $20.3,20.4,127.3,128.1,128.6$, 129.0, 129.8, 140.1, 140.8, 142.4; MS (ESI) $\mathrm{m} / \mathrm{z} 234(\mathrm{M}+\mathrm{H})^{+}$; HRMS (ESI) Calcd for $\mathrm{C}_{16} \mathrm{H}_{14} \mathrm{~N}_{2}(\mathrm{M}+\mathrm{H})^{+} 234.1220$, found 234.1223.

(3d) 2-(3-nitrophenyl)quinoxaline: Light orange colour solid; mp 185$187^{\circ} \mathrm{C}$; ${ }^{1} \mathrm{H}$ NMR $\left(300 \mathrm{MHz}, \mathrm{CDCl}_{3}\right) \delta(\mathrm{ppm})$ 7.72-7.90 (m, 3H, Ar-H), 8.11$8.25(\mathrm{~m}, 2 \mathrm{H}, \mathrm{Ar}-\mathrm{H}), 8.30(\mathrm{td}, 1 \mathrm{H}, J(1,2)=8.3, J(1,3)=2.3, J(1,3)=1.5,, \mathrm{Ar}-\mathrm{H})$, 8.6(d, $1 \mathrm{H}, J=8.3, \mathrm{Ar}-\mathrm{H}), 9.09-9.15(\mathrm{~m}, 1 \mathrm{H}, \mathrm{Ar}-\mathrm{H}), 9.41(\mathrm{~s}, 1 \mathrm{H}, \mathrm{Ar}-\mathrm{H}) ;{ }^{13} \mathrm{C} \mathrm{NMR}$ $\left(\mathrm{CDCl}_{3}, 75 \mathrm{MHz}\right) \delta(\mathrm{ppm}) 122.5,124.7,129.2,129.8,130.2,130.5,130.9$, 133.1, 142.5; MS (ESI) m/z: $252(\mathrm{M}+\mathrm{H})^{+}$; HRMS (ESI) Calcd for $\mathrm{C}_{14} \mathrm{H}_{9} \mathrm{~N}_{3} \mathrm{O}_{2}$ $(\mathrm{M}+\mathrm{H})^{+} 252.1652$ found 252.1657 .

(3e) 3-(quinoxalin-2-yl) benzenamine: Yellow colour solid; mp $163-$ $165^{\circ} \mathrm{C}$; ${ }^{1} \mathrm{H}$ NMR $\left(500 \mathrm{MHz}, \mathrm{CDCl}_{3}\right) \delta(\mathrm{ppm}) 3.80(\mathrm{bs}, 2 \mathrm{H},-\mathrm{NH} 2), 6.76(\mathrm{dd}$, $1 \mathrm{H}, J(1,2)=2.9, J(1,3)=8.9, \mathrm{Ar}-\mathrm{H}), 7.20-7.33(\mathrm{~m}, 1 \mathrm{H}, \mathrm{Ar}-\mathrm{H}), 7.45-7.59(\mathrm{~m}, 2 \mathrm{H}$, Ar-H), 7.64-7.80(m, 2H, Ar-H), 8.09(s, 2H, J=10.9, Ar-H), 9.25 (s, 1H, Ar-H); ${ }^{13} \mathrm{C}$ NMR $\left(\mathrm{CDCl}_{3}, 75 \mathrm{MHz}\right) \delta(\mathrm{ppm}) 113.8,117.0,117.8,129.1,129.4,129.5$, $130.1,130.2,137.8,141.6,142.2,143.5,147.3,152.0 ; \mathrm{MS}$ (ESI) $\mathrm{m} / \mathrm{z}: 222$ $(\mathrm{M}+\mathrm{H})^{+}$; HRMS (ESI) Caled for $\mathrm{C}_{16} \mathrm{H}_{11} \mathrm{~N}_{3}(\mathrm{M}+\mathrm{H})^{+} 222.1249$, found 222.1258 .

(3f) 6-methyl-2-p-tolylquinoxaline: Milky white colour solid; mp 146$147^{\circ} \mathrm{C} ;{ }^{1} \mathrm{H}$ NMR $\left(300 \mathrm{MHz}, \mathrm{CDCl}_{3}\right) \delta(\mathrm{ppm}) 2,45\left(\mathrm{~s}, 3 \mathrm{H}, \mathrm{Ar}-\mathrm{CH}_{3}\right), 2.61(\mathrm{~s}, 3 \mathrm{H}$, Ar- $\left.\mathrm{CH}_{3}\right), 7.31(\mathrm{~d}, J=8.3,3 \mathrm{H}, \mathrm{Ar}-\mathrm{H}), 7.59-7.47(\mathrm{~m}, 1 \mathrm{H}, \mathrm{Ar}-\mathrm{H}), 8.02-7.79(\mathrm{~m}$, $2 \mathrm{H}, \mathrm{Ar}-\mathrm{H}), 8.07$ (d, $J=8.3,2 \mathrm{H}, \mathrm{Ar}-\mathrm{H}), 9.22$ (d, $J=7.5,1 \mathrm{H}, \mathrm{Ar}-\mathrm{H}) ;{ }^{13} \mathrm{C} \mathrm{NMR}$ $\left(\mathrm{CDCl}_{3}, 75 \mathrm{MHz}\right) \delta(\mathrm{ppm}) 21.3,128.7,129.1,129.2,129.3,129.4,130.3,142.7$, 142.8, 150.9; MS (ESI) m/z $234(\mathrm{M}+\mathrm{H})^{+}$; HRMS (ESI) Calcd for $\mathrm{C}_{16} \mathrm{H}_{14} \mathrm{~N}_{2}$ $(\mathrm{M}+\mathrm{H})^{+} 234.1230$, found 234.1237 .

(3g) 6,7-dimethyl-2-p-tolylquinoxaline: Light brown colour solid; mp $127-129^{\circ} \mathrm{C} ;{ }^{1} \mathrm{H}$ NMR $\left(300 \mathrm{MHz}, \mathrm{CDCl}_{3}\right) \delta$ (ppm) 2,45 (s, 3H, Ar-CH3), 2.51 (s, 6H, Ar-CH3), 7.30 (d, $J=8.0,2 \mathrm{H}, \mathrm{Ar}-\mathrm{H}), 7.82$ (d, $J=9.5,2 \mathrm{H}, \mathrm{Ar}-\mathrm{H}), 8.05$ (d, $J=8.0,2 \mathrm{H}, \mathrm{Ar}-\mathrm{H}), 9.17$ (s, $1 \mathrm{H}, \mathrm{Ar}-\mathrm{H}) ;{ }^{13} \mathrm{C}$ NMR $\left(75 \mathrm{MHz}, \mathrm{CDCl}_{3}\right) \delta(\mathrm{ppm})$ 21.3, 22.9, 128.5, 129.0, 129.1, 129.3, 139.9, 140.3, 141.3, 142.9, 151.9; MS (ESI) $\mathrm{m} / \mathrm{z} 248(\mathrm{M}+\mathrm{H})^{+}$; HRMS (ESI) Calcd for $\mathrm{C}_{16} \mathrm{H}_{14} \mathrm{~N}_{2}(\mathrm{M}+\mathrm{H})^{+} 248.1390$, found 248.1398 .

(3h) 2-p-tolylquinoxaline: Brown colour solid; mp 90-92 ${ }^{\circ} \mathrm{C}$; ${ }^{1} \mathrm{H}$ NMR ( $\left.300 \mathrm{MHz}, \mathrm{CDCl}_{3}\right) \delta(\mathrm{ppm}) 2.45$ (s, 3H, Ar- $\left.\mathrm{CH}_{3}\right), 7.37-7.29$ (m, 2H, Ar-H), 7.77-7.64 (m, 2H, Ar-H), 8.14-8.04 (m, 4H, Ar-H), $9.28(\mathrm{~s}, 1 \mathrm{H}, \mathrm{Ar}-\mathrm{H}) ;{ }^{13} \mathrm{C}$ $\operatorname{NMR}\left(75 \mathrm{MHz}, \mathrm{CDCl}_{3}\right) \delta(\mathrm{ppm}) 21.4,127.4,129.0,129.2,129.5,129.8,130.1$, 133.9, 140.4, 141.4, 142.2, 143.2, 151.7; MS (ESI) m/z: $220(\mathrm{M}+\mathrm{H})^{+}$; HRMS (ESI) Calcd for $\mathrm{C}_{15} \mathrm{H}_{12} \mathrm{~N}_{2}(\mathrm{M}+\mathrm{H})^{+} 220.1080$, found 220.1083 .

(3i) 2-(4-fluorophenyl) quinoxaline: ${ }^{16}$ Yellow colour solid; mp: 120$122^{\circ} \mathrm{C}$; ${ }^{1} \mathrm{H}$ NMR (JCAMP, $\mathrm{CDCl}_{3}$ ) $\delta$ (ppm) 7.18-7.28 (m, 2H, Ar-H), 7.677.82 (m, 2H, Ar-H), 8.06-8.12 (td, $2 \mathrm{H}, \mathrm{Ar}-\mathrm{H}), 8.18-8.27$ (m, 2H, Ar-H), 9.27 $(\mathrm{s}, 1 \mathrm{H}, \mathrm{Ar}-\mathrm{H}) ;{ }^{13} \mathrm{C}$ NMR $\left(75 \mathrm{MHz}, \mathrm{CDCl}_{3}\right) \delta(\mathrm{ppm}) 116.0,116.3,129.1,129.4$, 129.5, 129.5, 130.3, 132.9, 142.8, 150.6, 165.8; MS (ESI) m/z $225(\mathrm{M}+\mathrm{H})^{+}$; HRMS (ESI) Calcd for $\mathrm{C}_{14} \mathrm{H}_{9} \mathrm{FN}_{2}(\mathrm{M}+\mathrm{H})^{+} 225.0924$, found 225.0929 .

(3j) 2-(4-nitro-phenyl)-quinoxaline: Yellow colour solid; mp $187-189^{\circ} \mathrm{C}$; ${ }^{1} \mathrm{H}$ NMR (400 MHz, $\left.\mathrm{CDCl}_{3}\right) \delta(\mathrm{ppm})$ 7.77-7.85 (m, 2H, Ar-H), 8.11-8.18 (m, $2 \mathrm{H}, \mathrm{Ar}-\mathrm{H}), 8.40$ (s, 4H, Ar-H), 9.36 (s, 1H, Ar-H); ${ }^{13} \mathrm{C} \mathrm{NMR} \mathrm{(75} \mathrm{MHz,} \mathrm{CDCl}_{3}$ ) $\delta(\mathrm{ppm}) 127.7,128.4,129.2,130.1,130.8,131.4,133.0,138.6,142.8,147.0$, 149.1; MS (ESI) $\mathrm{m} / \mathrm{z} 252(\mathrm{M}+\mathrm{H})^{+}$; HRMS (ESI) Calcd for $\mathrm{C}_{14} \mathrm{H}_{9} \mathrm{~N}_{3} \mathrm{O}_{2}(\mathrm{M}+\mathrm{H})^{+}$ 252.0756 , found 252.0766 .

(3k) 4-quinoxalin-2-yl-aniline: Black colour solid; mp $167-169^{\circ} \mathrm{C} ;{ }^{1} \mathrm{H}$ $\operatorname{NMR}\left(500 \mathrm{MHz}, \mathrm{CDCl}_{3}\right) \delta(\mathrm{ppm}) 6.76\left(\mathrm{dd}, 1 \mathrm{H}, J_{(12)}=8.9, J_{(13)}=3.0, \mathrm{Ar}-\mathrm{H}\right)$, 7.29 (t, $1 \mathrm{H}, J=7.9, \mathrm{Ar}-\mathrm{H}), 7.45-7.58(\mathrm{~m}, 2 \mathrm{H}), 7.64-7.79(\mathrm{~m}, 2 \mathrm{H}, \mathrm{Ar}-\mathrm{H}), 8.09$ $(\mathrm{t}, 2 \mathrm{H}, J=10.8, \mathrm{Ar}-\mathrm{H}), 9.25(\mathrm{~s}, 1 \mathrm{H}, \mathrm{Ar}-\mathrm{H}) ;{ }^{13} \mathrm{C} \mathrm{NMR}\left(75 \mathrm{MHz}, \mathrm{CDCl}_{3}\right) \delta(\mathrm{ppm})$ $113.7,116.9,117.6,129.0,129.3,129.4,129.9,130.1,137.7,141.4,142.1$, 143.4, 147.2; MS (ESI) m/z $222(\mathrm{M}+\mathrm{H})^{+}$; HRMS (ESI) Calcd for $\mathrm{C}_{16} \mathrm{H}_{11} \mathrm{~N}_{3}$ $(\mathrm{M}+\mathrm{H})^{+} 222.1392$, found 242.1410 .

(3I) 2-(6-chloropyridin-3-yl)quinoxaline: White solid; M.P; $135-137^{\circ} \mathrm{C} ;{ }^{1} \mathrm{H}$ NMR (300 MHz, $\left.\mathrm{CDCl}_{3}\right): d 9.32(\mathrm{~s}, 1 \mathrm{H}), 9.19(\mathrm{~d}, 1 \mathrm{H}, J=2.26 \mathrm{~Hz}), 8.49-8.53$ $(\mathrm{dd}, 1 \mathrm{H}, J=2.2,5.28 \mathrm{~Hz}), 8.16-8.10(\mathrm{~m}, 2 \mathrm{H}), 7.85-7.76(\mathrm{~m}, 2 \mathrm{H}), 7.51(\mathrm{~d}, 1 \mathrm{H}$, $J=8.3 \mathrm{~Hz}$ ); IR (KBr): 2978, 2853, 1624, 1580, 1476, 1364, 1208, 1104, 946 $\mathrm{cm}^{-1} ;$ EIMS: $m / z:(\mathrm{M}+\mathrm{H})^{+}: 242.0$

(3m) 2-(6-chloropyridin-3-yl)-7-methylquinoxaline: White solid; M.P; 151-153 ${ }^{\circ} \mathrm{C} ;{ }^{1} \mathrm{HbNMR}$ (300 MHz, CDCl +DMSO): $d 9.33(\mathrm{~d}, 2 \mathrm{H}, \mathrm{J}=5.12$ ), $9.21(\mathrm{~d}, 2 \mathrm{H}, J=2.92 \mathrm{~Hz}), 8.61-8.54$ (dd, $2 \mathrm{H}, J=2.9,5.8 \mathrm{~Hz}$ ), 8.04-7.95 (dd, $2 \mathrm{H}, J=2.9,5.8 \mathrm{~Hz}), 7.92-7.86(\mathrm{~m}, 2 \mathrm{H}), 7.68-7.58(\mathrm{~m}, 2 \mathrm{H}), 7.53(\mathrm{~d}, 2 \mathrm{H}, J=8.7$ Hz), 2.65 (s, 6H); IR (KBr): 2920, 2853, 1872, 1630, 1580, 1436, 1322, 1210 , 1104, 1007, $870 \mathrm{~cm}^{-1}$; EIMS: $m / z:(\mathrm{M}+\mathrm{H})^{+}: 256.0$.

(3n) 2-(6-chloropyridin-3-yl)-6,7-dimethylquinoxaline: Colourless solid; M.P; $170-172^{\circ} \mathrm{C} ;{ }^{1} \mathrm{H}$ NMR $\left(300 \mathrm{MHz}, \mathrm{CDCl}_{3}\right): d 9.22(\mathrm{~s}, 1 \mathrm{H}), 9.18(\mathrm{~d}, 1 \mathrm{H}$, $J=2.2 \mathrm{~Hz}), 8.49-8.53(\mathrm{dd}, 1 \mathrm{H}, J=2.2,6.0 \mathrm{~Hz}), \mathrm{d} 7.88(\mathrm{~d}, 2 \mathrm{H}, J=3.7 \mathrm{~Hz})$, $7.51(\mathrm{~d}, 1 \mathrm{H}, J=8.3 \mathrm{~Hz}), 2.57(\mathrm{~s}, 6 \mathrm{H}) ;{ }^{13} \mathrm{C}$ NMR $\left(75 \mathrm{MHz}, \mathrm{CDCl}_{3}\right): d 152.6$, $148.2,147.0,141.4,141.2,140.9,137.1,131.6,128.5,128.1,124.5,116.2$,
29.6, 20.3; IR (KBr): 2978, 2920, 2853, 1624, 1580, 1528, 1476, 1436, 1364 , 1322, 1208, 1104, $1052 \mathrm{~cm}^{-1}$; ESIMS: $\mathrm{m} / z$ : $(\mathrm{M}+\mathrm{H})^{+}:$270.0; HRMS calculated for $\mathrm{C}_{15} \mathrm{H}_{12} \mathrm{ClN}_{3}$ 270.0798. Found. 270.0790.

(3o) Brown colour solid. mp 92-94 ${ }^{\circ} \mathrm{C}$; ${ }^{1} \mathrm{H}$ NMR $\left(300 \mathrm{MHz}, \mathrm{CDCl}_{3}\right) \delta$ (ppm) 6.65- $6.79(\mathrm{~m}, 3 \mathrm{H}, \mathrm{Ar}-\mathrm{H}), 7.08-7.20(\mathrm{~m}, 2 \mathrm{H}, \mathrm{Ar}-\mathrm{H}), 7.65-7.80(\mathrm{~m}, 2 \mathrm{H}$, Ar-H), 8.05-8.13 (m, 2H, Ar-H), 8.16- 8.25 (m, 2H, Ar-H), 9.27 (s, 1H, Ar$\mathrm{H}) ;{ }^{13} \mathrm{C}$ NMR $\left(300 \mathrm{MHz}, \mathrm{CDCl}_{3}\right) \delta(\mathrm{ppm}) 96.1,107.7,108.0,110.5,110.8$, $119.6,120.9,127.9,128.0,128.2,129.2,129.4,129.5,129.6,129.7,130.1$, 130.2, 142.5, 156.7, 166.8; MS (ESI) $\mathrm{m} / z 362(\mathrm{M}+\mathrm{H})^{+}$; HRMS(ESI) Calcd for $\mathrm{C}_{20} \mathrm{H}_{12} \mathrm{FN}_{3} \mathrm{O}_{3}(\mathrm{M}+\mathrm{H})^{+}$362.0940, found: 362.0926 .

\section{ACKNOWLEDGEMENTS}

Financial supports from Council of Scientific and Industrial Research and Indian Institute of Chemical Technology are gratefully acknowledged.

\section{REFERENCES}

1. Ali M. M., Ismail M. M. F., EI-Gabby M. S. A., Zahran M. A., Ammar T. A., Molecules, 5, 864, (2000).

2. Sarges R., Howard H. R., Browne R. C., Label L. A., Seymour P. A., J. Med. Chem., 33, 2240, (1990).

3. Sakata G., Makino K., Kurasawa Y., Heterocycles, 27, 2481, (1998).

4. Arthur G., Elor K. B., Robert G. S., Guo Z. Z., Richard J. P., Stanley D., John R. K., Sean T., J. Med. Chem., 48, 744, (2005).

5. Singh, S. K.; Saibaba, V.; Ravikumar, V.; Rudrawar, S. V.; Daga, P.; Rao, C. S.; Akhila, V.; Hegde, P.; Rao, Y. K. Bioorg. Med. Chem. 12, 1881, (2004).

6. Katoh, A.; Yoshida, T.; Ohkanda, J. Heterocycles, 52, 911, (2000).

7. Thomas, K. R. J.; Velusamy, M.; Lin, J. T.; Chuen, C.-H.; Tao, Y.-T. Chem. Mater., 17, 1860, (2005).

8. Dailey, S.; Feast, W. J.; Peace, R. J.; Sage, I. C.; Till, S.; Wood, E. L. J. Mater. Chem. 11, 2238, (2001).

9. Sascha, O.; Ru“ diger, F. Synlett 1509, (2004).

10. Sessler, J. L.; Maeda, H.; Mizuno, T.; Lynch, V. M.; Furuta, H. J. Am. Chem. Soc. 124, 13474, (2002)

11. Crossley, M. J.; Johnston, L. A. Chem. Commun. 1122, (2002).

12. Dell, A.; William, D. H.; Morris, H. R.; Smith, G. A.; Feeney, J.; Roberts, G. C. K. J.

Am. Chem. Soc. 97, 2497, (1975)

13. Bailly, C.; Echepare, S.; Gago, F.; Waring, M. J. Anti-Cancer Drug Des. 15, 291, (1999).

14. Raw, S. A.; Wilfred, C. D.; Taylor, R. J. K. Org. Biomol. Chem. 2, 788, (2004).

15. Aparicio, D.; Attanasi, O. A.; Filippone, P.; Ignacio, R.; Lillini, S.; Mantellini, F.;

Palacios, F.; de los Santos, J. M. J. Org. Chem. 71, 5897, (2006).

16. Singh, S. K.; Gupta, P.; Duggineni, S.; Kundu, B. Synlett 2147, (2003).

17. Antoniotti, S.; Dun ach, E. Tetrahedron Lett. 43, 3971, (2002).

18. Mao, L. Sakurai, H. Hirao, T. Synthesis 2535, (2004).

19. Zhao, Z.; Wisnoski, D. D.; Wolkenberg, S. E.; Leister, W. H.; Wang, Y.; Lindsley, C.

W. Tetrahedron Lett. 45, 4873, (2004).

20. Jain N, Kumar A, Chauhan S \& Chauhan, Tetrahedron 61, 1015, (2005)

21. Galinski M, Lewandowski A \& Stepniak I, Electrochimica Acta 51, 5567, (2006).

22. R. M. Cowper and L. H. Davidson. Organic Syntheses, 5, 117, (2005). 